\title{
High Low-Density Lipoprotein Cholesterol Levels are Associated with Osteoporosis Among Adults 20-59 Years of Age
}

\author{
Ruijie Xie $\mathbb{D}^{\prime}$, Xiongjie Huang', Ya Zhang ${ }^{2}$, Qianlong Liu, Mingjiang Liu' \\ 'Department of Hand Surgery, The Affiliated Nanhua Hospital, Hengyang Medical School, University of South China, Hengyang, 42I002, People's \\ Republic of China; ${ }^{2}$ Affiliated Hospital of Youjiang Medical University for Nationalities, Baise, 533099, People's Republic of China; ${ }^{3}$ The Affiliated \\ Changsha Central Hospital, Hengyang Medical school, University of South China, Changsha, 410004, People's Republic of China
}

Correspondence: Mingjiang Liu, Email usclmj@I63.com

\begin{abstract}
Background: Serum lipids are highly inheritable and play a major role in bone health. However, the relationship between low-density lipoprotein cholesterol (LDL-C) and bone mineral density (BMD) remains uncertain. The goal of this study was to see if there was a link between LDL-C levels and BMD in persons aged 20 to 59.

Methods: Using data from the National Health and Nutrition Examination Survey (NHANES) 2011-2018, multivariate logistic regression models were utilized to investigate the association between LDL-C and lumbar BMD. Fitted smoothing curves and generalized additive models were also used.

Results: The analysis included a total of 4909 adults. After controlling for various variables, we discovered that LDL-C was negatively linked with lumbar BMD. The favorable connection of LDL-C with lumbar BMD was maintained in subgroup analyses stratified by gender and race in both males and females, Whites and Mexican Americans, but not in Blacks and other races. The relationship between LDL-C and lumbar BMD in other races was an inverted U-shaped curve with the inflection point: 2.327 (mmol/L).

Conclusion: In people aged 20 to 59, our research discovered a negative relationship among LDL-C and lumbar BMD. Among races other than Whites, Blacks, Mexican Americans, this relationship followed an inverted U-shaped curve (inflection point: $2.327 \mathrm{mmol} / \mathrm{L}$ ). LDL-C measurement might be used as a responsive biomarker for detecting osteoporosis early and guiding therapy.
\end{abstract}

Keywords: low-density lipoprotein cholesterol, bone mineral density, osteoporosis, osteopenia

\section{Background}

Osteoporosis and cardiovascular diseases (CVDs) are two major public health issues around the world. ${ }^{1,2}$ In North America, Europe, Japan, and Australia, osteoporosis affects up to 49 million individuals. CVDs caused approximately 17.8 million deaths worldwide in 2017. ${ }^{3,4}$ Both osteoporosis and cardiovascular diseases increase the risk of morbidity and mortality. ${ }^{5,6}$

A high level of low-density lipoprotein cholesterol (LDL-C) has been thought to be the major cause of atherosclerotic cardiovascular disease progression and death for decades. ${ }^{7}$ A number of studies have conclusively demonstrated that reducing plasma LDL-C levels using lipid-lowering medications is linked to a decreased risk of cardiovascular disease development and mortality. ${ }^{8-12}$

Osteoporosis is a long-term disorder marked by reduced bone mineral density (BMD) that affects a huge number of people. ${ }^{13}$ According to the International Osteoporosis Foundation, more than $30 \%$ of women and more than $20 \%$ of men over the age of 50 have osteoporosis or osteopenia, putting them at risk for osteoporotic fractures. ${ }^{14}$ Simultaneously, the prevalence of osteoporosis continues to climb as the population ages and expands. ${ }^{15}$ Apart from genetics, age, and gender, other variables that affect bone metabolisms, such as lipid metabolism and lifestyle, have lately received a lot of attention. ${ }^{16-18}$ Meanwhile, scientists are working to discover novel ways to prevent and treat osteoporosis. 
Lipids are now known to have an important role in bone metabolism, according to several research. ${ }^{19-21}$ For instance, Li et al, revealed that statin medication therapy helps enhance BMD by reducing LDL-C levels. ${ }^{20}$ Statins can also improve whole-body BMD, according to Zheng et al, which is linked to a reduction in LDL-C. ${ }^{21}$ Furthermore, whether HDL-C levels are linked to BMD is unknown and controversial. Many previous studies have looked at the relationship between LDL-C and BMD, however the results have been mixed. The associations between LDL- C and BMD were positive, ${ }^{22}$ adverse, ${ }^{23,24}$ and null. ${ }^{25}$ Since a result, it's worth investigating the relationship between LDL-C levels and BMD to see if LDL-C levels may be used to predict the likelihood of osteoporosis or osteopenia, as this might give a novel theoretical framework for understanding the genesis of the disease and designing therapies. As a result, we assessed the connection between LDL-C and BMD in this study using a comprehensive fraction of individuals aged 20 to 59 from the National Health and Nutrition Examination Survey (NHANES).

\section{Materials and Methods}

\section{Data Source and Study Population}

The NHANES is a major, continuing cross-sectional survey in the United States that aims to give objective statistics on health issues and address emerging public health concerns among the general public. The NHANES datasets were utilized for this investigation from 2011 to 2018. The participants in the research had to be between the ages of 20 and 59. Among the 39,156 eligible adults, we excluded 20,384 individuals with missing BMD data, 11,872 with missing LDL-C, 1813 individuals younger than 20 years or older than 59 years, and 178 individuals with cancer diagnoses. In the end, 4909 people were enrolled in the study.

\section{Ethics Statement}

The National Center for Health Statistics Research Ethics Review Board authorized the protocols for the NHANES and got signed informed consent. The protocols for this study were approved by the research ethics board at the affiliated Nanhua Hospital, University of South China. The NHANES data is accessible to the public after anonymization. This allows researchers to convert data into a format that can be studied. We agree to adhere to data usage constraints in this study to ensure that data is only used for statistical analysis and that all experiments are conducted in accordance with applicable standards and laws.

\section{Study Variables}

The levels of plasma cholesterol in patients who were tested in the morning were assessed. LDL-C is computed using Friedwald's formula using total cholesterol, high-density lipoprotein cholesterol (HDL-C), and triglyceride measurements: $[$ LDL-C $]=[$ total cholesterol $]-[$ HDL-C $]-[$ triglycerides/5]. Dual-energy X-ray absorptiometry was performed using a Hologic QDR 4500A device and Apex software version 3.2 by qualified radiology technologists to assess lumbar BMD. Covariates in multivariate models may cause the correlations between HDL-C and lumbar BMD to be muddled. Age, gender, race, educational level, BMI, family income-to-poverty ratio, moderate activities, smoking at least 100 cigarettes over the life period to the point of data collection, diabetes status, hypertension status, ALT, AST, Total calcium, Blood urea nitrogen, Serum uric acid, and Serum phosphorus were all covariates in this study. The NHANES website (https://www.cdc.gov/nchs/nhanes/) has a thorough explanation of how these variables are calculated.

\section{Statistical Analysis}

In order to account for our data set's high volatility, we used a weighted and variance estimation methodology. The relationship between LDL-C and lumbar BMD was investigated using a weighted multivariate logistic regression model. To calculate the difference between each group, we utilized the weighted 2 test for categorical variables and the weighted linear regression model for continuous variables. The subgroup analysis was carried out using stratified multivariate regression analysis. The nonlinear link between LDL-C and lumbar BMD was also addressed using smooth curve fits and generalized additive models. When nonlinearity was discovered, a recursive technique was utilized to determine the inflection point in the relationship between LDL-C and BMD, which was then followed by a two-piecewise linear 
regression model on both sides of the inflection point. All analyses were conducted using PackageR (http://www. r-project.org) and EmpowerStats (http://www.empowerstats.com), with a P value of 0.05 considered statistically significant.

\section{Results}

The individuals' weighted characteristics were divided into quartiles based on their HDL-C levels (Q1:0.62-2.30mmol/L; Q2: 2.31-2.85mmol/L; Q3: 2.86-3.47mmol/L; and Q4: 3.48-9.70mmol/L), as shown in Table 1. Our study included a total of 4909 people between the ages of 20 and 59. Between the LDL-C quartiles, there were substantial variations in baseline characteristics. Individuals in the top HDL-C quartile were much likely to be male, with higher BMI, Education level, Income to poverty ratio, ALT, Blood urea nitrogen, and serum uric acid, and lower lumbar BMD when compared to the other categories.

Table I Headers: Weighted Characteristics of the Study Population Based on LDL-C Quartiles

\begin{tabular}{|c|c|c|c|c|c|}
\hline LDL Cholesterol (mmol/L) & $\begin{array}{c}\text { Q I } \\
(\leq 2.30)\end{array}$ & $\begin{array}{c}\text { Q2 } \\
(2.3 \mid-2.85)\end{array}$ & $\begin{array}{c}\text { Q3 } \\
(2.86-3.47)\end{array}$ & $\begin{array}{c}\text { Q4 } \\
(\geq 3.48)\end{array}$ & $P$ value \\
\hline Age (years) & $35.286 \pm 11.87 \mid$ & $37.916 \pm 11.836$ & $40.934 \pm 11.287$ & $42.773 \pm 10.288$ & $<0.001$ \\
\hline Sex, n (\%) & & & & & 0.002 \\
\hline Male & 49.030 & 51.110 & 55.688 & 55.195 & \\
\hline Female & 50.970 & 48.890 & 44.312 & 44.805 & \\
\hline Race/Ethnicity (\%) & & & & & 0.215 \\
\hline Non-Hispanic White & 61.004 & 61.577 & 61.003 & 61.501 & \\
\hline Non-Hispanic Black & 13.082 & 10.987 & 9.931 & 11.573 & \\
\hline Mexican American & 9.469 & 10.535 & 11.725 & 8.957 & \\
\hline Other race/ethnicity & 16.444 & 16.900 & $|7.34|$ & 17.969 & \\
\hline Education level (\%) & & & & & 0.027 \\
\hline Less than high School & 12.268 & 15.335 & 14.623 & 14.618 & \\
\hline High school & 21.551 & 19.464 & 21.946 & 24.817 & \\
\hline More than high school & 66.180 & 65.201 & 63.431 & 60.566 & \\
\hline Body mass index $(\mathrm{kg} / \mathrm{m} 2)$ & $27.817 \pm 7.300$ & $28.381 \pm 6.737$ & $29.730 \pm 7.039$ & $29.880 \pm 6.360$ & $<0.001$ \\
\hline Income to poverty ratio & $2.736 \pm 1.659$ & $2.852 \pm 1.684$ & $2.945 \pm 1.653$ & $2.961 \pm 1.659$ & 0.004 \\
\hline Moderate activities (\%) & & & & & 0.128 \\
\hline Yes & 67.552 & 70.188 & 67.875 & 71.108 & \\
\hline No & 32.478 & 29.812 & 32.125 & 28.892 & \\
\hline Smoked at least 100 cigarettes in life, $n$ (\%) & & & & & 0.016 \\
\hline Yes & 37.042 & 42.720 & 43.835 & 43.067 & \\
\hline No & 62.958 & 57.280 & 56.164 & 56.934 & \\
\hline Diabetes, n (\%) & & & & & $<0.001$ \\
\hline Yes & 8.398 & 4.757 & 4.842 & 4.793 & \\
\hline No & 91.602 & 95.243 & 95.158 & 95.207 & \\
\hline Hypertension, n (\%) & & & & & 0.008 \\
\hline Yes & 20.168 & 24.007 & 22.053 & 25.740 & \\
\hline No & 79.832 & 75.993 & 77.947 & 74.260 & \\
\hline $\operatorname{ALT}(\mathrm{U} / \mathrm{L}$, mean $\pm \mathrm{SD})$ & $23.560 \pm 17.527$ & $24.611 \pm 18.728$ & $26.768 \pm 19.367$ & $28.540 \pm 18.5397$ & $<0.001$ \\
\hline AST $(U / L$, mean $\pm S D)$ & $25.362 \pm 29.638$ & $23.928 \pm 14.428$ & $24.539 \pm 14.252$ & $25.810 \pm 14.514$ & 0.073 \\
\hline Total calcium (mg/dL, mean $\pm \mathrm{SD}$ ) & $2.322 \pm 0.084$ & $2.328 \pm 0.079$ & $2.327 \pm 0.081$ & $2.347 \pm 0.080$ & $<0.001$ \\
\hline Blood urea nitrogen $(\mathrm{mg} / \mathrm{dL}$, mean $\pm \mathrm{SD})$ & $4.456 \pm 1.709$ & $4.490 \pm 1.529$ & $4.600 \pm 1.474$ & $4.630 \pm 1.373$ & 0.012 \\
\hline Serum uric acid (umol/L, mean $\pm S D$ ) & $304.736 \pm 77.466$ & $3|9.17| \pm 80.286$ & $325.209 \pm 80.402$ & $336.686 \pm 80.714$ & $<0.001$ \\
\hline Serum phosphorus $(\mathrm{mg} / \mathrm{dL}$, mean $\pm \mathrm{SD}$ ) & $1.191 \pm 0.180$ & $1.167 \pm 0.172$ & $1.169 \pm 0.179$ & $1.171 \pm 0.165$ & 0.002 \\
\hline Lumbar BMD $(\mathrm{g} / \mathrm{cm} 2$, mean $\pm \mathrm{SD})$ & $1.050 \pm 0.147$ & $1.038 \pm 0.146$ & $1.026 \pm 0.143$ & $1.014 \pm 0.140$ & $<0.001$ \\
\hline
\end{tabular}

Notes: Mean $\pm S D$ for continuous variables: the $P$ value was calculated by the weighted linear regression model. (\%) for categorical variables: the $P$ value was calculated by the weighted chi-square test.

Abbreviation: BMD, bone mineral density. 
Table 2 Headers: The Association Between Low-Density Lipoprotein Cholesterol (Mmol/L) and Lumbar Bone Mineral Density (g/cm2)

\begin{tabular}{|c|c|c|c|}
\hline & MODEL I $\beta(95 \% \mathrm{Cl})$ & MODEL $2 \beta(95 \% \mathrm{Cl})$ & MODEL $3 \beta(95 \% \mathrm{Cl})$ \\
\hline & $P$ value & $P$ value & $P$ value \\
\hline LDL cholesterol & $-0.0163(-0.0208,-0.0118)<0.001$ & $-0.0129(-0.0175,-0.0083)<0.001$ & $-0.0130(-0.0202,-0.0058)<0.001$ \\
\hline Quintiles of LDL cholesterol & & & \\
\hline QI & Reference & Reference & Reference \\
\hline Q2 & $-0.0115(-0.0229,-0.0001)$ & $-0.0070(-0.0182,0.0042)$ & $0.0064(-0.0112,0.0239)$ \\
\hline & 0.048306 & 0.219844 & 0.478176 \\
\hline Q3 & $-0.0239(-0.0353,-0.0124)$ & $-0.0150(-0.0264,-0.0036)$ & $-0.0069(-0.0249,0.0111)$ \\
\hline & 0.000043 & 0.009689 & 0.454672 \\
\hline Q4 & $-0.0359(-0.0473,-0.0245)$ & $-0.0278(-0.0393,-0.0163)$ & $-0.0226(-0.0413,-0.0039)$ \\
\hline & $<0.000001$ & 0.000002 & 0.017786 \\
\hline$P$ for trend & $<0.001$ & $<0.001$ & 0.010 \\
\hline Stratified by gender & & & \\
\hline Men & $-0.0168(-0.0234,-0.0102)$ & $-0.0153(-0.0218,-0.0088)$ & $-0.0165(-0.0267,-0.0064)$ \\
\hline & $<0.000001$ & 0.000004 & 0.001464 \\
\hline Women & $-0.0153(-0.0215,-0.0090)$ & $-0.0094(-0.0159,-0.0029)$ & $-0.0073(-0.0178,0.0031)$ \\
\hline Stratified by race & 0.000002 & 0.004567 & 0.170317 \\
\hline Non-Hispanic White & $-0.0136(-0.0210,-0.0062)$ & $-0.0110(-0.0186,-0.0034)$ & $-0.0082(-0.0194,0.0029)$ \\
\hline & 0.000325 & 0.004588 & 0.147490 \\
\hline Non-Hispanic Black & $-0.0232(-0.034 I,-0.0123)$ & $-0.0190(-0.0301,-0.0079)$ & $-0.016 \mathrm{I}(-0.035 \mathrm{I}, 0.0028)$ \\
\hline & 0.000035 & 0.000854 & 0.096327 \\
\hline Mexican American & $-0.0198(-0.0303,-0.0092)$ & $-0.0171(-0.0282,-0.0060)$ & $-0.0218(-0.0394,-0.0042)$ \\
\hline & 0.000257 & 0.002553 & 0.016050 \\
\hline Other Race & $-0.0165(-0.0249,-0.0081)$ & $-0.0145(-0.0232,-0.0058)$ & $-0.0192(-0.0340,-0.0045)$ \\
\hline & 0.000124 & 0.001094 & 0.011017 \\
\hline
\end{tabular}

Notes: Model I: no covariates were adjusted. Model 2: age, gender, and race were adjusted. Model 3: age, gender, race, educational level, BMI, family income-to-poverty ratio, moderate activities, smoking at least I00 cigarettes over the life period to the point of data collection, diabetes status, hypertension status, ALT, AST, Total calcium, Blood urea nitrogen, Serum uric acid, and Serum phosphorus were adjusted. In the subgroup analysis stratified by gender and race, the model is not adjusted for sex and race, respectively. 
Table 3 Headers: Threshold Effect Analysis of Low-Density Lipoprotein Cholesterol on Lumbar Bone Mineral Density in Other Races Using the Two-Piecewise Linear Regression Model

\begin{tabular}{|l|c|}
\hline Lumbar Bone Mineral Density & $\begin{array}{c}\text { Adjusted } \boldsymbol{\beta}(\mathbf{9 5} \% \mathbf{C l}) \\
\boldsymbol{P} \text { value }\end{array}$ \\
\hline $\begin{array}{l}\text { Other races } \\
\text { Fitting by the standard linear model } \\
\text { Fitting by the two-piecewise linear } \\
\text { Model }\end{array}$ & $-0.019(-0.035,-0.003) 0.0213$ \\
Inflection point & \\
LDL cholesterol<2.327(mmol/L) & 2.327 \\
LDL cholesterol>2.327(mmol/L) & $0.063(0.008,0.119) 0.0254$ \\
Log likelihood ratio & $-0.038(-0.058,-0.018) 0.0002$ \\
\hline
\end{tabular}

Notes: Age, gender, educational level, BMI, family income-to-poverty ratio, moderate activities, smoking at least 100 cigarettes over the life period to the point of data collection, diabetes status, hypertension status, ALT, AST, Total calcium, Blood urea nitrogen, Serum uric acid, and Serum phosphorus were adjusted.

The findings of the multivariate regression analysis are shown in Table 2. LDL-C was negatively linked with lumbar BMD in the unadjusted model $(=-0.0163,95 \% \mathrm{CI}:-0.0208,-0.0118, \mathrm{P}<0.001)$. This significant correlation was remained apparent after adjusting for covariates in models $2(=-0.0129,95 \% \mathrm{CI}:-0.0175,-0.0083, \mathrm{P}<0.001)$ and 3 $(=-0.0130,95 \% \mathrm{CI}:-0.0202,-0.0058, \mathrm{P}<0.001)$. Individuals in the bottom LDL-C quartile had a $0.036 \mathrm{~g} / \mathrm{cm} 2 \mathrm{higher}$ BMD than those in the top LDL-C quartile after LDL-C has been converted from a continuous to a categorical variable (quartiles).

On a subgroup analysis stratified by gender, the negative correlation of LDL-C with lumbar BMD both maintained in men $(=-0.0165,95 \% \mathrm{CI}:-0.0267,-0.0064, \mathrm{P}=0.001)$, and in women $(=-0.0073,95 \% \mathrm{CI}:-0.0178,0.0031, \mathrm{P}=0.170)$, On a subgroup analysis stratified by race, the negative correlation as well as in Whites $(=-0.0082,95 \%$ CI: -0.0194 ,

A

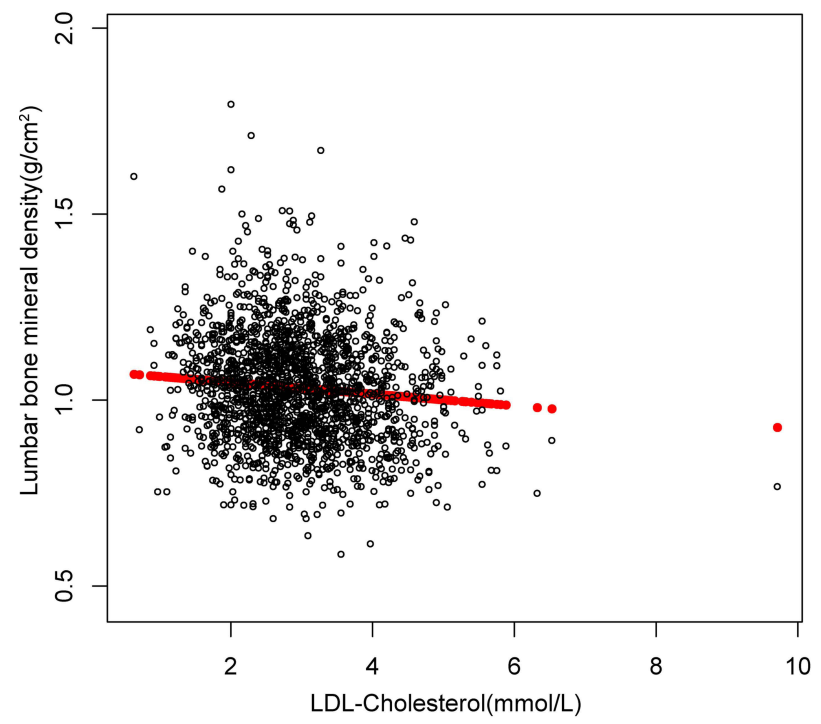

B

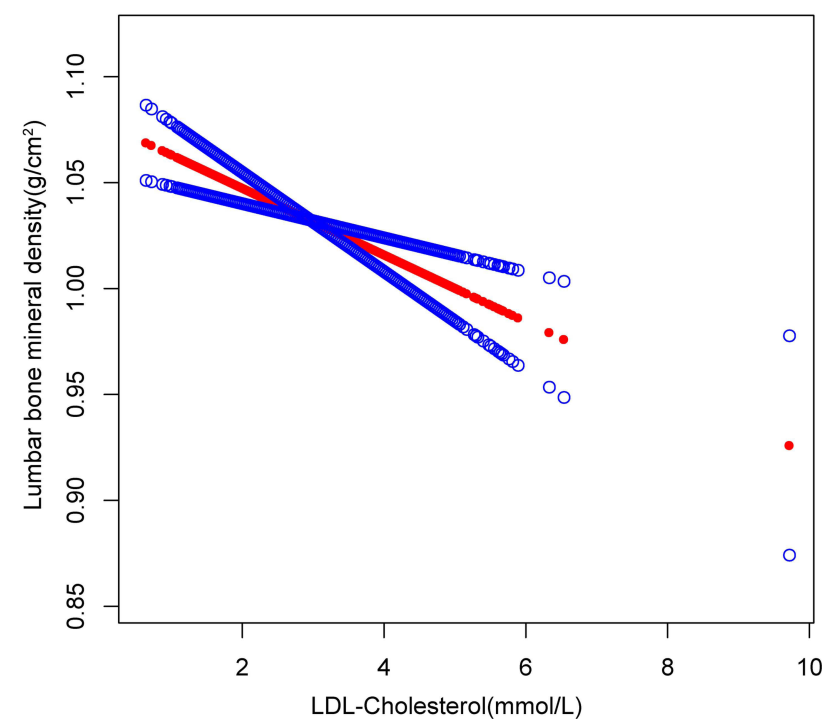

Figure I The association between low-density lipoprotein cholesterol and lumbar bone mineral density. (A) Each black point represents a sample. (B) Solid red line represents the smooth curve fit between variables. Blue bands represent the $95 \%$ of confidence interval from the fit. Age, gender, race, educational level, BMI, family incometo-poverty ratio, moderate activities, smoking at least 100 cigarettes over the life period to the point of data collection, diabetes status, hypertension status, ALT, AST, total calcium, blood urea nitrogen, serum uric acid, and serum phosphorus were adjusted. 


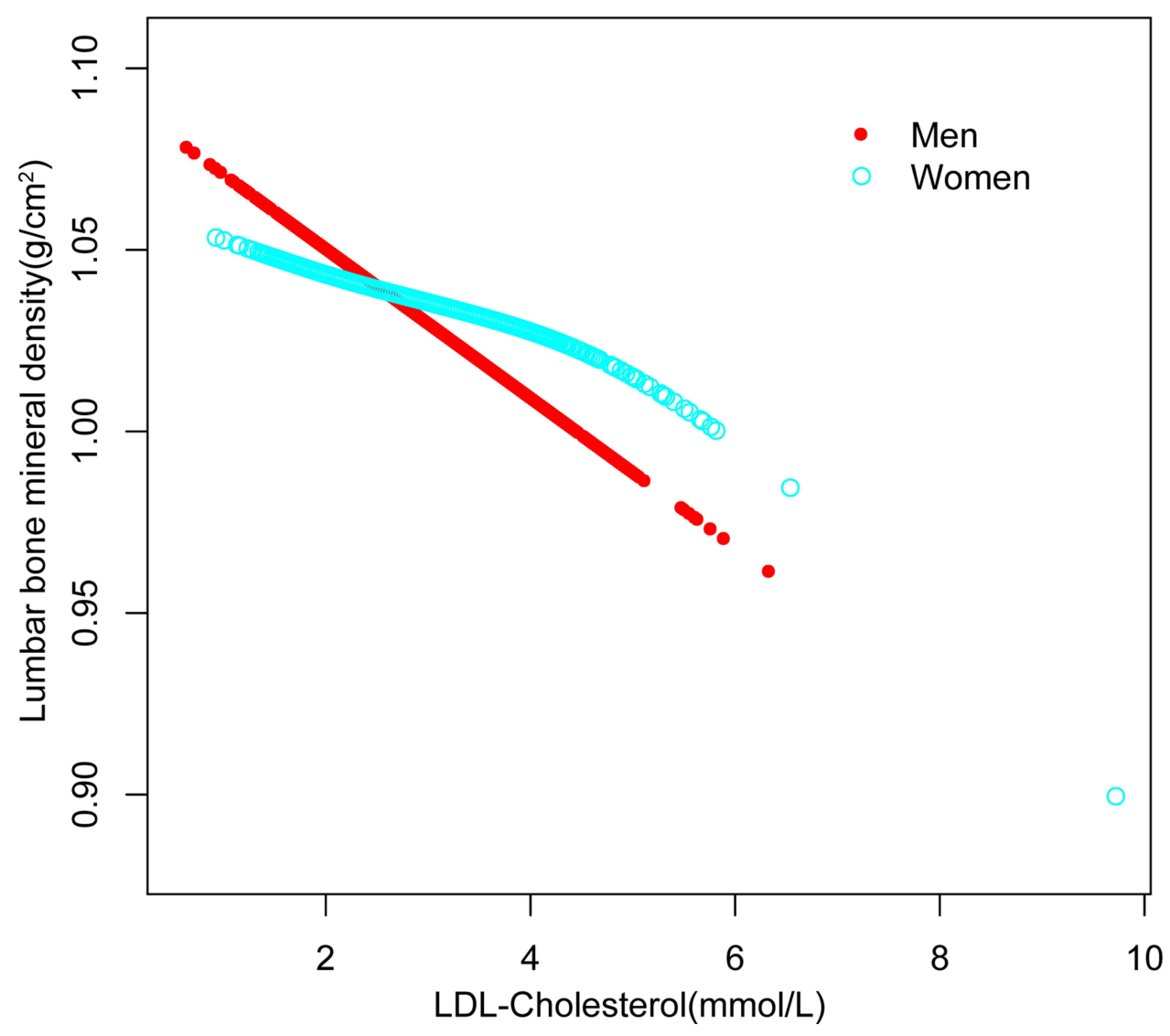

Figure 2 The association between low-density lipoprotein cholesterol and lumbar bone mineral density stratified by sex. Age, race, educational level, BMI, family income-topoverty ratio, moderate activities, smoking at least 100 cigarettes over the life period to the point of data collection, diabetes status, hypertension status, ALT, AST, total calcium, blood urea nitrogen, serum uric acid, and serum phosphorus were adjusted.

0.0029, $P=0.147)$, and Mexican American $(=-0.0218,95 \%$ CI: $-0.0394,-0.0042, P=0.016)$, but not in Blacks $(=$ $-0.0161,95 \%$ CI: $-0.0351,0.0028, \mathrm{P}=0.096)$, and other races $(=-0.0192,95 \% \mathrm{CI}:-0.0340,-0.0045, \mathrm{P}=0.011)$. Figures 1- Figure 3 depict smooth curve fits and generalized additive models that were utilized to define the nonlinear connection among LDL-C and lumbar BMD. The relationship among LDL-C and lumbar BMD in other races was an inverted U-shaped curve at $2.327(\mathrm{mmol} / \mathrm{L})$ for HDL-C. (Table 3).

\section{Discussion}

Our multivariate logistic regression analysis revealed that a higher LDL-C level was linked with a lower lumbar BMD in the current research. On subgroup analysis, however, we discovered an inverted U-shaped relationship among LDL-C and lumbar BMD in races other than Whites, Blacks, Mexican Americans.

Clinical investigations on the connection between LDL-C and BMD in older persons are still few and disputed. In the Hong Kong Osteoporosis Study $(n=1128)$ and a US representative cohort NHANES III $(n=3638)$, researchers discovered a negative relationship among LDL-C and BMD. ${ }^{20}$ Other research from the UK and China, ${ }^{26,27}$ backed up this result. However, other studies contradicted this finding. A cross-sectional research conducted in China found a favorable connection between LDL-C and BMD in females $(n=1116){ }^{28}$ Research from Spain found that in heavy alcoholism, BMD was directly connected to total cholesterol and LDL cholesterol. ${ }^{22}$ Furthermore, a cross-sectional research with 481 Chinese seniors found no evidence of a causal relationship between HDL-C and BMD at various locations. $^{25}$

The current study not only shows a link between LDL-C and BMD, but it also has therapeutic implications that can help doctors. The negative correlation shows that people with a lower LDL-C level may also have a higher BMD. We also ran subgroup analysis in our research for a more accurate depiction of the data set. Our findings suggest that in both 


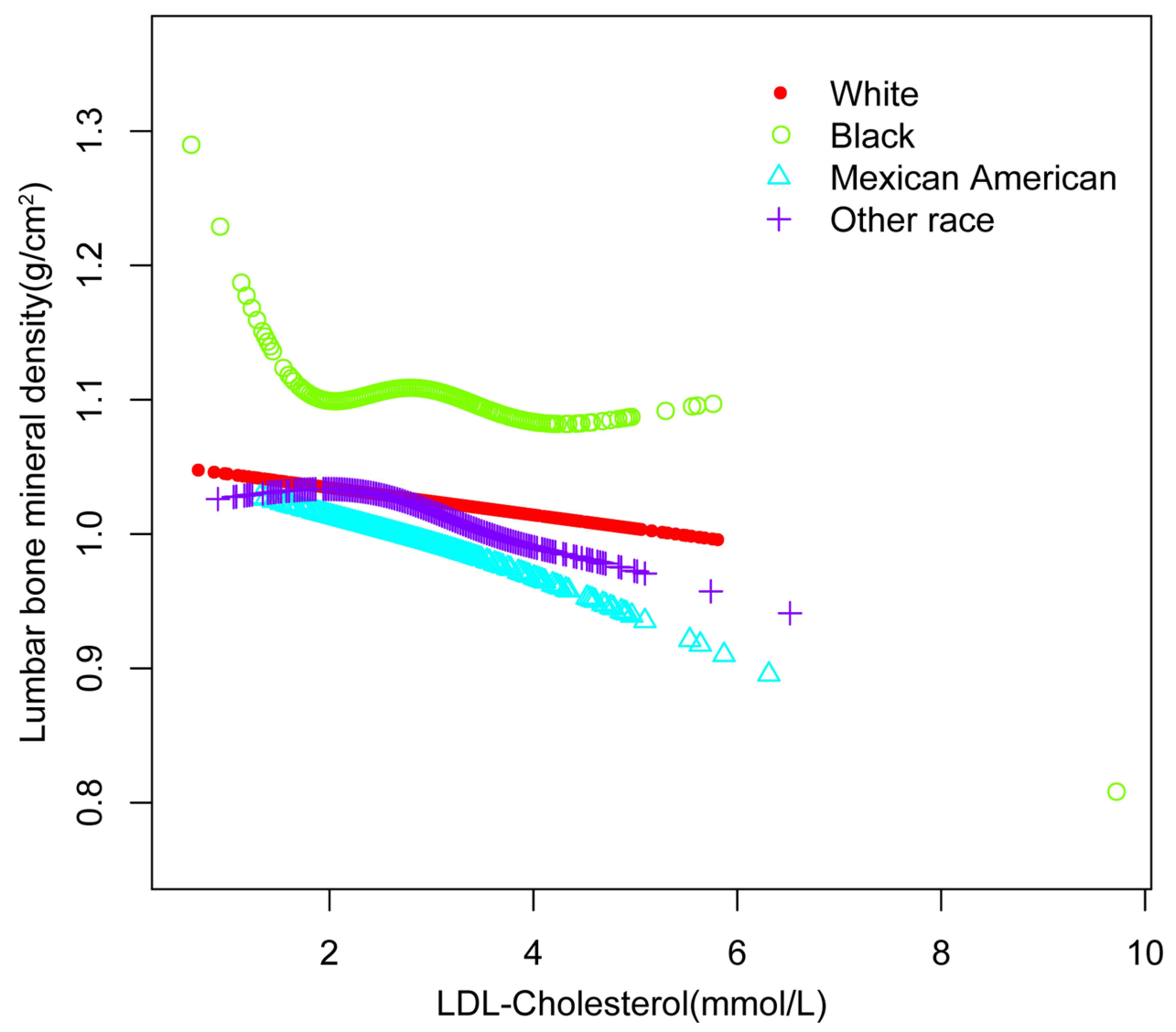

Figure 3 The association between low-density lipoprotein cholesterol and lumbar bone mineral density stratified by race. Age, gender, educational level, BMI, family incometo-poverty ratio, moderate activities, smoking at least 100 cigarettes over the life period to the point of data collection, diabetes status, hypertension status, ALT, AST, total calcium, blood urea nitrogen, serum uric acid, and serum phosphorus were adjusted.

men and women, and also Whites and Mexican Americans, lower LDL-C is associated with higher lumbar BMD. But in Blacks, LDL-C and BMD are non-linearly related. And in other races, there is an inverted U-shaped relationship among LDL-C and BMD, when LDL-C is greater than 2.327 (mmol/L), the relationship among HDL-C levels and lumbar BMD is positively correlated, when LDL-C is less than $2.327(\mathrm{mmol} / \mathrm{L})$, the relationship is negative. To further understand the link between HDL-C and BMD by gender and race, more prospective studies with large study populations are needed.

The involvement of LDL-C in bone metabolism was explained mechanistically using animal and cell experiments. In a mouse model, ${ }^{29}$ a high-cholesterol diet lowered BMD, most likely because of increased osteoclastogenesis. ${ }^{30}$ Statins, on the other hand, target the mevalonate pathway, which is also targeted by the first-line medication for osteoporosis, nitrogen-containing bisphosphonates (N-BPs). In ovariectomized rats, ${ }^{31}$ statin treatment decreased bone loss by inhibiting osteoclastogenesis and increased osteoblast development in ovariectomized rabbits. ${ }^{32}$ Statins have been shown to reduce bone resorption by blocking osteoclast development and osteoblast apoptosis downstream of the mevalonate pathway, ${ }^{33}$ which might explain why statins' lowering of LDL-C may have an extra favorable impact on BMD.

Postmenopausal women have been the focus of the majority of cohort and cross-sectional research so far. The link between LDL-C and BMD in young healthy people is poorly known. The findings of our study are extremely applicable to the entire population since we selected a nationally comprehensive sample. Furthermore, because of our large sample size, we were able to conduct subgroup analyses of LDL-C and lumbar BMD in people of different genders and races. However, it is critical to recognize the study's limitations. The cross-sectional methodology of our investigation, first and foremost, restricts the inference of a causal relationship between LDL-C and lumbar BMD in adults. More large sample prospective studies and fundamental mechanistic research are needed to understand the particular mechanism of the link among LDL-C and BMD. Second, malignancy patients were excluded from the research cause cancer might have a big impact on lumbar BMD. Third, bone health is considered to be influenced by sex hormones, but information on sex 
hormone levels was not accessible or absent from the NHANES database 2011-2018, our study was unable to explain these situations in the present patients.

\section{Conclusion}

In people aged 20 to 59, our research discovered a negative association among LDL-C and lumbar BMD. Among races other than Whites, Blacks, Mexican Americans, this relationship followed an inverted U-shaped curve (inflection point: $2.327 \mathrm{mmol} / \mathrm{L}$ ). LDL-C measurement might be used as a responsive biomarker for detecting osteoporosis early and guiding therapy.

\section{Abbreviations}

LDL-C, low-density lipoproteins cholesterol; HDL-C, high-density lipoprotein cholesterol; CVDs, cardiovascular diseases; BMD, bone mineral density; NHANES, National Health and Nutrition Examination Survey; nitrogen-containing bisphosphonates - (N-BPs).

\section{Data Sharing Statement}

The survey data are publicly available on the internet for data users and researchers throughout the world (www.cdc.gov/ nchs/nhanes/).

\section{Ethical Statement}

The protocols for the conduct of NHANES was approved by National Center for Health Statistics Research Ethics Review Board and written informed consent was obtained. The protocols for this study were approved by the research ethics board at the Affiliated Nanhua Hospital, University of South China.

\section{Acknowledgments}

We thank the National Health and Nutrition Examination Surveys for providing the data.

\section{Author Contributions}

All authors made a significant contribution to the work reported, whether that is in the conception, study design, execution, acquisition of data, analysis and interpretation, or in all these areas; took part in drafting, revising or critically reviewing the article; gave final approval of the version to be published; have agreed on the journal to which the article has been submitted; and agree to be accountable for all aspects of the work.

\section{Funding}

This study was funded by the Scientific Research Project of Hunan Health and Family Planning Commission (A2017018).

\section{Disclosure}

The authors declare that they have no competing interests.

\section{References}

1. Compston J, McClung M, Leslie W. Osteoporosis. Lancet. 2019;393(10169):364-376. doi:10.1016/S0140-6736(18)32112-3

2. Laslett L, Alagona P, Clark B, et al. The worldwide environment of cardiovascular disease: prevalence, diagnosis, therapy, and policy issues: a report from the American College of Cardiology. J Am Coll Cardiol. 2012;60:S1-49. doi:10.1016/j.jacc.2012.11.002

3. Wade S, Strader C, Fitzpatrick L, Anthony M, O’Malley C. Estimating prevalence of osteoporosis: examples from industrialized countries. Arch Osteoporos. 2014;9:182. doi:10.1007/s11657-014-0182-3

4. Kyu HH. Global, regional, and national disability-adjusted life-years (DALYs) for 359 diseases and injuries and healthy life expectancy (HALE) for 195 countries and territories, 1990-2017: a systematic analysis for the Global Burden of Disease Study 2017. Lancet. 2018;392(10159):1859-1922. doi:10.1016/S0140-6736(18)32335-3

5. Liu S, Li Y, Zeng X, et al. Burden of Cardiovascular Diseases in China, 1990-2016: findings From the 2016 Global Burden of Disease Study. JAMA Cardiology. 2019;4(4):342-352. doi:10.1001/jamacardio.2019.0295 
6. Yu F, Xia W. The epidemiology of osteoporosis, associated fragility fractures, and management gap in China. Arch Osteoporos. 2019;14(1):32. doi:10.1007/s11657-018-0549-y

7. Lewington S, Whitlock G, Clarke R, et al. Blood cholesterol and vascular mortality by age, sex, and blood pressure: a meta-analysis of individual data from 61 prospective studies with 55,000 vascular deaths. Lancet. 2007;370(9602):1829-1839.

8. Benn M, Tybjærg-Hansen A, Stender S, Frikke-Schmidt R, Nordestgaard B. Low-density lipoprotein cholesterol and the risk of cancer: a Mendelian randomization study. J Natl Cancer Inst. 2011;103(6):508-519. doi:10.1093/jnci/djr008

9. Jacobs D, Blackburn H, Higgins M, et al. Report of the Conference on Low Blood Cholesterol: mortality Associations. Circulation. 1992;86 (3):1046-1060. doi:10.1161/01.CIR.86.3.1046

10. Linsel-Nitschke P, Götz A, Erdmann J, et al. Lifelong reduction of LDL-cholesterol related to a common variant in the LDL-receptor gene decreases the risk of coronary artery disease-a Mendelian Randomisation study. PLoS One. 2008;3(8):e2986. doi:10.1371/journal.pone.0002986

11. Neaton J, Blackburn H, Jacobs D, et al. Serum cholesterol level and mortality findings for men screened in the Multiple Risk Factor Intervention Trial. Multiple Risk Factor Intervention Trial Research Group. Arch Intern Med. 1992;152(7):1490-1500. doi:10.1001/ archinte.1992.00400190110021

12. Strasak A, Pfeiffer R, Brant L, et al. Time-dependent association of total serum cholesterol and cancer incidence in a cohort of 172,210 men and women: a prospective 19-year follow-up study. Ann Oncol. 2009;20(6):1113-1120. doi:10.1093/annonc/mdn736

13. Ensrud K, Crandall C. Osteoporosis. Ann Intern Med. 2017;167(3):ITC17-ITC32. doi:10.7326/AITC201708010

14. Wright N, Looker A, Saag K, et al. The recent prevalence of osteoporosis and low bone mass in the United States based on bone mineral density at the femoral neck or lumbar spine. J Bone Mineral Res. 2014;29(11):2520-2526. doi:10.1002/jbmr.2269

15. Alejandro P, Constantinescu F. A Review of Osteoporosis in the Older Adult: an Update. Rheum Dis Clin North Am. 2018;44(3):437-451. doi:10.1016/j.rdc.2018.03.004

16. Nomura S, Kitami A, Takao-Kawabata R, et al. Teriparatide Improves Bone and Lipid Metabolism in a Male Rat Model of Type 2 Diabetes Mellitus. Endocrinology. 2019;160(10):2339-2352. doi:10.1210/en.2019-00239

17. Gajewska J, Weker H, Ambroszkiewicz J, et al. Alterations in markers of bone metabolism and adipokines following a 3-month lifestyle intervention induced weight loss in obese prepubertal children. Exp Clin Endocrinol Diabetes. 2013;121(8):498-504. doi:10.1055/s-0033-1347198

18. Villareal D, Shah K, Banks M, Sinacore D, Klein S. Effect of weight loss and exercise therapy on bone metabolism and mass in obese older adults: a one-year randomized controlled trial. J Clin Endocrinol Metab. 2008;93(6):2181-2187. doi:10.1210/jc.2007-1473

19. Tian L, Yu X. Lipid metabolism disorders and bone dysfunction-interrelated and mutually regulated (review). Mol Med Rep. 2015;12(1):783-794. doi:10.3892/mmr.2015.3472

20. Li G, Cheung C, Au P, Tan K, Wong I, Sham P. Positive effects of low LDL-C and statins on bone mineral density: an integrated epidemiological observation analysis and Mendelian randomization study. Int J Epidemiol. 2020;49(4):1221-1235. doi:10.1093/ije/dyz145

21. Zheng J, Brion M, Kemp J, et al. The Effect of Plasma Lipids and Lipid-Lowering Interventions on Bone Mineral Density: a Mendelian Randomization Study. J Bone Mineral Res. 2020;35(7):1224-1235. doi:10.1002/jbmr.3989

22. Martín-González C, González-Reimers E, Quintero-Platt G, et al. Lipid profile and bone mineral density in heavy alcoholics. Clin Nutr. 2018;37:2137-2143. doi:10.1016/j.clnu.2017.10.008

23. Chen Y, Wang W, Yang L, Chen W, Zhang H. Association between lipid profiles and osteoporosis in postmenopausal women: a meta-analysis. Eur Rev Med Pharmacol Sci. 2018;22(1):1-9. doi:10.26355/eurrev_201801_14093

24. Andersen L, Ibarra J, Andersen R. Current familial hypercholesterolemia diagnostic criteria underdiagnose APOB mutations: lessons from the Amish community. J Clin Lipidol. 2016;10(2):443-444. doi:10.1016/j.jacl.2015.11.013

25. Kan B, Zhao Q, Wang L, Xue S, Cai H, Yang S. Association between lipid biomarkers and osteoporosis: a cross-sectional study. BMC Musculoskelet Disord. 2021;22(1):759. doi:10.1186/s12891-021-04643-5

26. Cherny S, Freidin M, Williams F, Livshits G. The analysis of causal relationships between blood lipid levels and BMD. PLoS One. 2019;14(2): e0212464. doi:10.1371/journal.pone.0212464

27. Yang X, Cui Z, Zhang $\mathrm{H}$, et al. Causal link between lipid profile and bone mineral density: a Mendelian randomization study. Bone. 2019;127:37-43. doi:10.1016/j.bone.2019.05.037

28. Zhang Q, Zhou J, Wang Q, et al. Association Between Bone Mineral Density and Lipid Profile in Chinese Women. Clin Interv Aging. 2020;15:1649-1664. doi:10.2147/CIA.S266722

29. Pelton K, Krieder J, Joiner D, Freeman M, Goldstein S, Solomon K. Hypercholesterolemia promotes an osteoporotic phenotype. Am J Pathol. 2012;181(3):928-936. doi:10.1016/j.ajpath.2012.05.034

30. Sanbe T, Tomofuji T, Ekuni D, Azuma T, Tamaki N, Yamamoto T. Oral administration of vitamin C prevents alveolar bone resorption induced by high dietary cholesterol in rats. $J$ Periodontol. 2007;78(11):2165-2170. doi:10.1902/jop.2007.070181

31. Oxlund H, Andreassen T. Simvastatin treatment partially prevents ovariectomy-induced bone loss while increasing cortical bone formation. Bone. 2004;34(4):609-618. doi:10.1016/j.bone.2003.12.014

32. Zhou H, Xie Y, Baloch Z, Shi Q, Huo Q, Ma T. The effect of atorvastatin, 3-hydroxy-3-methylglutaryl coenzyme A reductase inhibitor (HMG-CoA), on the prevention of osteoporosis in ovariectomized rabbits. J Bone Miner Metab. 2017;35(3):245-254. doi:10.1007/s00774-016$0750-2$

33. Ruan F, Zheng Q, Wang J. Mechanisms of bone anabolism regulated by statins. Biosci Rep. 2012;32(6):511-519. doi:10.1042/BSR20110118 


\section{Publish your work in this journal}

The International Journal of General Medicine is an international, peer-reviewed open-access journal that focuses on general and internal medicine, pathogenesis, epidemiology, diagnosis, monitoring and treatment protocols. The journal is characterized by the rapid reporting of reviews, original research and clinical studies across all disease areas. The manuscript management system is completely online and includes a very quick and fair peer-review system, which is all easy to use. Visit http://www.dovepress.com/testimonials.php to read real quotes from published authors.

Submit your manuscript here: https://www.dovepress.com/international-journal-of-general-medicine-journal 